\title{
Effet des constituants pariétaux sur la capacité d'ingestion et sur le comportement alimentaire de la vache laitière
}

\author{
V Girard *, J Labonté
}

\begin{abstract}
Service de la zootechnie, Direction de la recherche et du développement, ministère de l'Agriculture, des Pêcheries et de l'Alimentation, Québec, Canada
\end{abstract}

(Reçu le 7 juillet 1992; accepté le 20 juillet 1993)

\begin{abstract}
Résumé - Quatre vaches $(568,5 \pm 17,9 \mathrm{~kg})$ produisant $23,8 \pm 3,9 \mathrm{~kg}$ de lait sont utilisées pour mesurer l'effet d'une modification des constituants pariétaux insolubles dans un détergent neutre (NDF) sur la capacité d'ingestion de matière sèche (MS) et sur la dynamique du comportement alimentaire. Du jour 1 au jour 17 de chaque période, les vaches reçoivent une ration témoin composée de $50 \mathrm{~kg}$ d'ensilage de fléole à $41 \%$ de MS avec $24 \%$ d'orge et de graines de soya entière. Á partir du jour 17 et jusqu'à la fin de la période ( $\mathrm{j} 23$ ), les vaches reçoivent, selon un dispositif en carré latin $2 \times 2$, la ration témoin ou la ration témoin $+4,4 \mathrm{~kg}$ de pulpe de papier (traitement $T+N D F$ ). Le comportement alimentaire de chaque vache est enregistré à chaque minute du jour 14 au jour 17 (phase 1) et du jour 20 au jour 23 (phase 2). En moyenne journalière pendant la phase 1, les vaches ont consommé $23,2 \pm 1,6 \mathrm{~kg}$ de MS contenant $10 \pm 0,5 \mathrm{~kg}$ de constituants pariétaux NDF avec un temps d'ingestion de $290 \pm 28$ minutes, avec $34500 \pm 8300$ coups de mâchoire pendant la mastication mérycique (MM) et $3150 \pm 1183 \mathrm{MM}$ par kg de constituants pariétaux NDF. La capacité d'ingestion varie d'une vache à l'autre $(P<0,01)$, ainsi que MM $(P<0,05)$ et $\mathrm{MM}$ par $\mathrm{kg}$ de constituants pariétaux NDF $(P<0,05)$, mais pendant la phase 1 , il n'y a aucune corrélation $(P>0,05)$ entre la mastication et la capacité d'ingestion. Pendant la phase 2, la capacité d'ingestion diminue avec $\mathrm{MM}(r=0,76$, $P<0,01)$. Le traitement $T+$ NDF réduit $(P<0,01)$ la capacité d'ingestion $(-10,3 \%)$ et MM $(-33 \%)$ mais l'ingestion de NDF n'est pas affectée. Pour étudier la dynamique du comportement alimentaire, un profil de $24 \mathrm{~h}$ est établi à l'aide de 12 observations répétées dans le temps et les profils individuels des 2 phases sont comparés. L'effet du temps de l'observation sur les différences entre profils est analysé avec les carrés latins et les phases comme effets principaux et le temps et ses interactions comme effets secondaires. Ces derniers sont décomposés en effets linéaires, quadratiques, etc, à l'aide de 11 contrastes polynomiaux. En regard de la ration témoin, le supplément NDF modifie l'aspect du profil de l'ingestion de MS et celui de la MM de façon linéaire avec le temps $(P<$ $0,01)$, tandis que la modification de l'ingestion de NDF est curviligne $(P<0,01)$. Lorsque la concentration en constituants pariétaux augmente, les vaches donnent davantage d'importance aux repas qui suivent la distribution d'aliment et réduisent le volume des repas qui précèdent la distribution d'aliment suivante.
\end{abstract}

ingestion / constituants pariétaux / observations répétées dans le temps / mastication

* Correspondance et tirés à part: Service de la zootechnie, 120-A, chemin du Roy, Deschambault, Québec, GOA 1SO, Canada. 
Summary - Effect of cell wall on voluntary intake and feeding behavior of lactating cows. Four Holstein cows $(568 \pm 17.9)$ in midlactation $(23.8 \pm 3.9 \mathrm{~kg}$ milk production) were used to test the effect of neutral detergent insoluble cell-wall fiber (NDF) on behavior and voluntary dry matter intake (DMI). The period length was $23 \mathrm{~d}$. Up to d 17, all cows were fed $50 \mathrm{~kg}$ timothy silage $(41 \% \mathrm{DM})$ with $24 \%$ barley and soya (control) and then allotted, according to a $2 \times 2$ latin square design, either to the control diet or to the control diet plus $4.4 \mathrm{~kg}$ NDF pulp. Feeding and chewing behaviors were recorded at 1 -min intervals between $d 14$ and d 17 (phase 1) and between d 20 and d 23 (phase 2). During phase 1 , all the cows were fed the control diet and ingested $23.2 \pm 1.6 \mathrm{~kg}$ with $10 \pm 0.5 \mathrm{~kg} \mathrm{NDF}$ in $290 \pm 28$ min. Cows chewed $34500 \pm 8300$ times during rumination (RUM) or $3150 \pm 1183 R U M \mathrm{~kg}^{-1} \mathrm{NDF}$. In phase 1, cows had different (P <0.01) DMI, RUM and RUM $\mathrm{kg}^{-1}$ NDF but DMI was not correlated with chewing. However, in phase 2, DMI variations were correlated $(\mathrm{r}=0.76, \mathrm{P}<0.01)$ with RUM. Feeding the NDF pulp decreased $(\mathrm{P}<0.01)$ DMI $(-10.3 \%)$ and RUM $(-33 \%)$ but not the NDF intake. The behavior over $24 \mathrm{~h}$ was condensed into 12 observations and individual behaviors were compared between phases. The time-response differences were analysed with the latin square and phase effects as main plots and observation times as subplots. Subplots were split into linear, quadratic and other effects of time by 11 polynomial contrasts. In regard to the control diet, the time-response difference caused by the NDF pulp was linear $(P<0.01)$ for DMI and RUM and quadratic $(P<0.01)$ for NDF intake. Cows fed the NDF pulp had higher DMI and NDF intake after feeding and spend less time eating and ruminating before feeding than cows fed the control diet.

\section{NDF intake / cell wall / time-response analysis / chewing activity}

\section{INTRODUCTION}

En Amérique du Nord, la capacité d'ingestion est définie comme étant la consommation ad libitum de constituants pariétaux insolubles dans un détergent neutre (NDF) (Mertens, 1987), tandis qu'en France elle est représentée par l'ingestion volontaire d'un fourrage standard distribué seul (Inra, 1978 ; Jarrige et al, 1986). Elle est stable lorsque la digestion et l'évacuation des particules non digérées sont en équilibre avec la prise alimentaire. Or, dans le rumen, c'est principalement la mastication qui, en réduisant la taille des particules alimentaires (McLeod et Minson, 1988), facilite la digestion et l'évacuation. On peut donc penser a priori que la mastication et la capacité d'ingestion sont liées et interagissent l'une sur l'autre.

La mastication peut avoir lieu pendant les repas (mastication ingestive) ou pendant la rumination (mastication mérycique). En général, chaque repas est suivi d'une période de rumination et la répartition de ces activités pendant $24 \mathrm{~h}$ consti- tue un profil du comportement alimentaire. D'un animal à un autre, les profils peuvent varier sans modifier la capacité d'ingestion (Beauchemin et Buchanan-Smith, 1989 ; Dulphy et al, 1990). Cela est particulièrement le cas pour la fréquence des repas (Charmley et al, 1991 ; Dehareng et Godeau, 1991) et la durée de la rumination (Deswysen et al, 1987 ; Dehareng et Godeau, 1991). Au contraire, les variations de la durée et de l'importance du repas principal à l'auge modifient la capacité d'ingestion (Norgaard, 1986; Deswysen et al, 1987 ; Beaumont et al, 1989 ; Moseley et Manendez, 1989 ; Francke et al, 1990). L'importance du repas principal à l'auge et la capacité d'ingestion sont réduites lorsqu'on déclenche de façon prématurée la rumination en stimulant la paroi ruminale (Beaumont et al, 1990).

La relation entre les constituants pariétaux NDF, la mastication et la capacité d'ingestion pourraient donc être envisagées comme un ajustement du stimulus qui déclenche la rumination et abrège la durée du repas principal. Dans un groupe 
de ruminants, l'intensité du stimulus causée par les constituants pariétaux peut être très variable. Mais il est intéressant de choisir l'individu comme son propre témoin, car sans modification des aliments, la variabilité inter-individuelle du comportement alimentaire est beaucoup plus grande que la variabilité intra-individuelle, au moins pendant 9 jours (Girard et Labonté, 1993).

Le présent travail aborde ce problème en étudiant les modifications du comportement alimentaire engendrées en introduisant dans le traitement expérimental un aliment broyé, mais plus riche en constituants pariétaux.

Notre étude du comportement repose sur des observations répétées dans le temps et l'objectif de cette présentation est de proposer un modèle statistique approprié.

\section{MATÉRIEL ET MÉTHODES}

Quatre vaches $(568,5 \pm 17,9 \mathrm{~kg})$ produisant 23,8 $\pm 3,9 \mathrm{~kg}$ de lait sont choisies au hasard dans un troupeau comprendant 24 vaches de race Hol- stein en mi-lactation. Elles sont placées dans des stalles individuelles équipées d'auges sur capteur de charge. Un contact magnétique installé sur les licols permet de compter les coups où la mâchoire inférieure est complètement relevée (Girard et Labonté, 1993), c'est-à-dire les coups de mâchoire où il y a broiement. Chaque jour, à $14 \mathrm{~h}$, les vaches reçoivent $50 \mathrm{~kg}$ d'une ration mélangée témoin (Témoin) contenant $73,6 \%$ d'ensilage de fléole récolté en début d'épiaison sans agent conservateur à $41 \%$ de matière sèche (MS), $12,4 \%$ d'orge, $12,8 \%$ de graines de soya entières et $0,6 \mathrm{~kg}(1,2 \%)$ d'un mélange de minéraux (tableau I). Les quantités d'aliment sont suffisantes pour assurer $10 \%$ de refus et l'eau est disponible en permanence. Les 4 vaches sont disposées en 2 carrés latins $2 \times 2$ (Carré) comprenant 2 périodes de $23 \mathrm{j}$ (Période), 2 traitements (Trait) et 2 colonnes (CoIonne). Chaque période comprend 2 phases (Phase), la première joue le rôle de valeur de base (fig 1) et est utilisée pour établir des comparaisons avec la phase suivante. $\dot{A}$ partir du $17^{\mathrm{e}}$ jour de chaque période, on distribue, mélangés à $50 \mathrm{~kg}$ de ration témoin, $4,4 \mathrm{~kg}$ de pulpe de papier délignifiée (NF105 Kraft, Weyerhaeuser, Sudburt, Canada; traitement T + NDF), soit un supplément équivalent à $3,8 \mathrm{~kg}$ de constituants pariétaux NDF. Ce supplément a modifié la qualité des constituants pariétaux dans la ration en réduisant la taille moyenne des particules fibreuses (tableau I).

Tableau I. Composition chimique des aliments (en $\mathrm{g} / \mathrm{kg} \mathrm{MS}$ excepté autres indications).

\begin{tabular}{|c|c|c|c|c|c|c|}
\hline & Ensilage & Orge & Soja & Pulpe papier & Témoin a & $\begin{array}{l}\text { Témoin }{ }^{\mathrm{a}}+ \\
\text { pulpe papier }\end{array}$ \\
\hline Matière sèche $(g / k g)$ & 410 & 860 & 920 & 820 & 535 & 559 \\
\hline Matière organique & 939 & 974 & 912 & 997 & 908 & 918 \\
\hline $\mathrm{N}$ total & 133 & 124 & 428 & 0 & 188 & 188 \\
\hline Constituants pariétaux NDF & 664 & 200 & 125 & 997 & 434 & 507 \\
\hline Constituants pariétaux ADF & 330 & 85 & 95 & 810 & 222 & 298 \\
\hline $\mathrm{N}-\mathrm{NH}_{3}(\% \mathrm{~N}$ total $)$ & $\begin{array}{l}1,5 \\
42\end{array}$ & 0 & 0 & 0 & & \\
\hline Longueur de la fibre $(\mathrm{cm})$ & 2,7 & & & 0,3 & $2,7^{b}$ & $2,3^{b}$ \\
\hline \multirow{2}{*}{\multicolumn{4}{|c|}{$\begin{array}{l}\text { NDF dégradable in situ après } 120 \mathrm{~h} \\
\text { Vitesse horaire de dégradation du NDF }\end{array}$}} & 996 & 655 & 740 \\
\hline & & & & 0,045 & 0,061 & 0,049 \\
\hline
\end{tabular}

a Avec $0,6 \mathrm{~kg}$ minéraux contenant $16 \%$ de calcium, $8,4 \%$ de phosphore, $5,2 \%$ de manganèse et $10,5 \%$ de sodium.

b Valeur calculée à partir des constituants. 


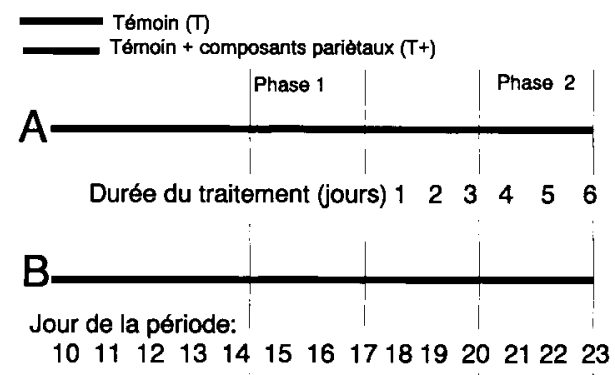

Fig 1. Dispositif expérimental en carré latin. Les premières colonnes de chaque carré, attribuées à $\mathbf{A}$ en première période, le sont à $\mathbf{B}$ en deuxième période, et vice-versa.

\section{Qualité des constituants pariétaux}

Des sachets en polyester $(5 \times 10 \mathrm{~cm}$, porosité $53 \mathrm{m \mu}$ ), préalablement pesés, sont remplis avec approximativement $2 \mathrm{~g}$ d'aliment moulu (ø 2 $\mathrm{mm}$ ) dans un cyclotec (\#1093, Tecator). Scellés à chaud, les sachets sont lyophilisés pendant $72 \mathrm{~h}$ et pesés puis incubés in situ en triple dans le rumen de 2 vaches recevant la ration témoin. On détermine la dégradabilité des constituants pariétaux des aliments après $12,24,48,72,96$ et $120 \mathrm{~h}$ d'incubation et un lavage de $2 \mathrm{~min}$ à l'eau froide. Par convention, les valeurs obtenues dans 3 sachets lavés sans incubation sont celles du temps zéro et toutes les autres valeurs sont rapportées en pourcentage du temps zéro. Les variations dans le temps des résidus de la dégradation des constituants pariétaux NDF ont été analysées avec un modèle de digestion différée (Van Milgen et al, 1991).

\section{Ingestion et rumination}

L'ingestion d'aliment et la mastication mérycique ne pouvant avoir lieu simultanément, l'examen des variations du poids de l'auge permet de distinguer la mastication mérycique de la mastication pendant l'ingestion. Lorsque les réductions du poids de l'auge sont supérieures à l'erreur de pesée, soit $150 \mathrm{~g}$ (Girard et Labonté, 1993), la mastication est considérée ingestive. Dans le cas contraire, la mastication est attribuée à la rumination si la fréquence des coups de mâchoires dépasse 35 coups de mâchoires $\mathrm{min}^{-1}$. La fréquence permet de distinguer la mastication mérycique des autres activités où la mâchoire inférieure est complètement relevée, c'est-à-dire les coups de langue isolés et la consommation d'eau.

\section{Profils journaliers, profils horaires et profils nycthéméraux}

Les 3 profils journaliers de chaque phase représentent $4332 \pm 40$ enregistrements, soit une observation toutes les minutes. Afin de condenser cette information, les profils journaliers sont d'abord exprimés à l'aide des 24 moyennes horaires (profil horaire, fig 2a). On procède ensuite à une seconde simplification en calculant le comportement moyen par tranche de $2 \mathrm{~h}$, soit 12 observations (fig 2b), qui constituent ce que nous appellerons le profil nycthéméral de la phase. Dans une précédente expérience (Girard et Labonté, 1993), nous avons démontré la faible variabilité intra-individuelle des profils nycthéméraux pendant des périodes de $9 \mathrm{j}$. Ainsi, l'observation $X_{1 k}^{i}$, c'est-à-dire la $i^{e}$ observation du profil nycthéméral de la première phase pendant la $k^{e}$ période et avec la le vache, peut servir de référence à l'observation $x_{2 \mathrm{kl}}^{i}$ (deuxième phase). Dans cette expérience, nous considérons le profil nycthéméral de la première phase comme un profil de référence.

\section{Validation des profils nycthéméraux}

Avant de procéder à l'analyse du comportement alimentaire nycthéméral, il est nécessaire d'évaluer l'erreur introduite en condensant l'information contenue dans plus de 4000 observations en 24 (profil horaire) ou 12 observations (profil nycthéméral). Nous avons choisi un modèle asymptotique :

$$
X_{k k l}^{i}=A_{j k \mid}{ }^{*}\left[1-\exp \left(-b_{j k l}{ }^{*} i\right)\right]
$$

(Baumont et al, 1989) pour décrire l'ingestion pendant les 8 premières heures qui suivent le début du premier repas. Les paramètres $A_{j k l}$ et $b_{j k l}$ ont été estimés à l'aide de la méthode Marquardt dans la procédure NLIN de SAS (1987). Dans un premier temps, 1440 observations sont utilisées, soit toutes les observations enre- 
a)

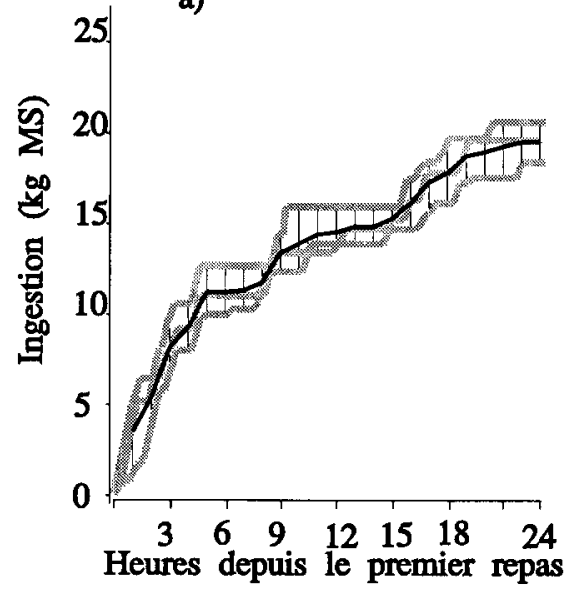

b)

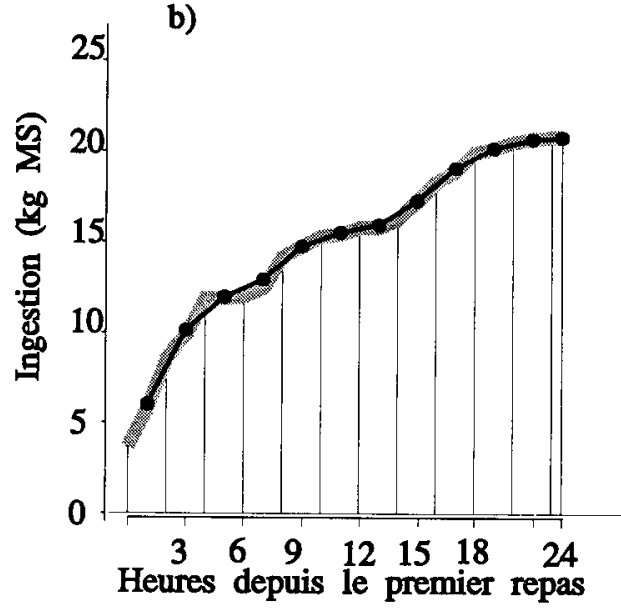

Fig 2. Profil horaire de l'ingestion ( $a \mathbf{a}$; $b$ ) et profil nycthéméral correspondant $(\mathbf{b} \boldsymbol{m})$ ). Le profil horaire décrit le comportement moyen horaire d'une vache observée toutes les minutes pendant $3 \mathrm{j}$ consécutifs (a axo:), c'est-à-dire une répétition. On procède ensuite à une simplification du profil journalier en calculant le comportement moyen par tranches de $2 \mathrm{~h}$, soit 12 observations ( $)$ qui constituent ce que nous appelons le profil nycthéméral de la répétition.

gistrées pendant les 8 premières heures de chacun des 3 profils journaliers. Puis les mêmes coefficients sont estimés à partir du profil horaire ( 8 observations), et finalement à partir du profil nycthéméral ( 4 ob-servations). On compare ensuite à l'aide d'un test de $T$, les $A_{j k !}$ et $b_{j k l}$ obtenus avec le profil horaire et nycthéméral par rapport aux valeurs obtenues avec toutes les observations. La variation des coefficients représente l'erreur introduite lorsqu'on réduit le nombre d'observation de 1440 à 8 puis à 4 et que l'on représente la dynamique de l'ingestion à l'aide du modèle de Baumont.

\section{Capacité d'ingestion}

Nous avons émis une hypothèse, à savoir que la mastication et la capacité d'ingestion, c'est-àdire la dernière observation du profil nycthéméral de l'ingestion de matière sèche ( $\left.\mid \mathrm{NG}_{j \mathrm{kl}}^{24 h}\right)$, sont lièes et interagissent l'une sur l'autre. La présence d'un lien est examinée séparément pendant la phase 1 et pendant la phase 2. Pendant la phase 1 , les coefficients de corrélation entre $\mid N G_{1 k l}^{24 h}$ et le début $\left(X_{1 k l}^{2 h}, X_{1 k l}^{4 h}\right)$ ou la fin
$\left(X_{1 \mathrm{kl}}^{24 \mathrm{~h}}\right)$ du profil nycthéméral décrivent la relation entre le comportement alimentaire et la capacité d'ingestion dans un échantillon de vaches qui recoivent la même alimentation. Dans cette étude, $X_{i k l}^{i}$ représente la dynamique de la mastication (ingestive ou mérycique), de la durée de la mastication, de l'ingestion de constituants pariétaux et de la mastication par $\mathrm{kg}$ de constituants pariétaux. Pour la phase 2, nous déterminons les modifications du comportement alimentaire par rapport au profil de référence, $R X_{k 1}^{i}$, en soustrayant des valeurs observées, $X_{2 k \mid}^{i}$, les valeurs $X_{k \mid}^{i}$ obtenues pendant la phase 1 . Les modifications du comportement en début et en fin de profil nycthéméral corrélées aux modifications de la capacité d'ingestion ( $R I N G_{k l}^{24 h}$ ) décrivent la relation entre la capacité d'ingestion et les constituants pariétaux NDF lorsque l'alimentation varie.

\section{Profils nycthéméraux de référence et variations dues aux traitements}

Nous avons examiné la dynamique des activités du comportement alimentaire corrélées 
significativement $(P<0,05)$ à la capacité d'ingestion.

Le profil nycthéméral de chacune des activités concernées $\left(X_{j k 1}^{i}\right)$ est analysé comme un dispositif en split-plot dans le temps, avec comme effets principaux l'effet des colonnes et celui des périodes dans chacun des carrés latins, soit Colonne(Carré) et Période(Carré) et l'effet des phases dans les traitements, soit Phase(Trait). L'effet Colonne(Carré) avec 3 degrés de liberté (d), représente les vaches. Dans l'effet Phase (Trait) (3 dl), un contraste permet de tester si les différences entre la phase 1 et la phase 2 varient selon les traitements (contraste $T$ vs $T$ + NDF). Le modèle comprend 2 types d'erreur $\varepsilon_{a}$ (7 dl) et $\varepsilon_{\mathrm{b}}(77 \mathrm{dl})$ :

$$
\begin{aligned}
& X_{i j k l}=\text { Colonne(Carré) }+ \text { Période(Carré) } \\
& + \text { Phase(Trait) }+\varepsilon_{a}+\text { Heure }_{i}+\text { Heure }_{i} \\
& \times \text { Colonne(Carré) }+ \text { Heure }_{1} \times \text { Période(Carré) } \\
& + \text { Heure }_{i} \times \text { Phase (Trait) }+\varepsilon_{b}
\end{aligned}
$$

Chaque profil comprenant 12 observations, l'erreur $\varepsilon_{b}$ contient 11 fois plus de degrés de liberté (dl) que l'erreur $\varepsilon_{\mathrm{a}}$. Mais dans cette analyse, l'effet des heures, les interactions des heures avec Colonne(Carré), Période(Carré), Phase(Trait) et le contraste T vs T + NDF, sont subdivisés en effets linéaires, quadratique, cubiques, etc, des heures à l'aide de 11 contrastes polynomiaux orthogonaux. Chacune des hypothèses (effet linéaire, quadratique, etc, de l'heure) est donc testée avec $7 \mathrm{dl}$ et l'erreur appropriée obtenue par la méthode de Rowell et Walters (1976) et la procédure GLM pour observations répétées (SAS, 1987).

\section{RÉSULTATS}

\section{Qualité des constituants pariétaux}

Tous les constituants pariétaux contenus dans la pulpe de papier délignifiée sont dégradables par les bactéries cellulolytiques du rumen, tandis que c'est le cas pour seulement $65,5 \%$ des constituants pariétaux de l'ensilage (tableau I). Par rapport au témoin, le traitement $T+N D F$ contient davantage de constituants pariétaux dégradables $(65,5 \%$ vs $72 \%)$ mais ceux-ci se dégradent moins vite (tableau I). En supposant un taux de passage horaire moyen de 3,7\% (Girard, 1990), la demi-vie dans le rumen (Girard, 1987) des constituants pariétaux du témoin est de $11,08 \mathrm{~h}$. Calculée de la même manière, la demi-vie des constituants pariétaux du traitement $T+$ NDF est de 11,03 h. La valeur d'encombrement des constituants pariétaux, basée sur la demi-vie des petites particules, est donc semblable dans les 2 traitements.

\section{Validation des profils nycthéméraux}

Les coefficients du modèle décrivant l'ingestion pendant les 8 premières heures de chaque période ont été calculés pour chacune des 2 phases par période et par vache, soit 16 estimés de $A_{j k l}$ et de $b_{j k l}$.

Lorsque toutes les observations des profils journaliers étaient utilisées (1 440 observations), $A_{j k l}$ a varié de 10,8 à $17,5 \mathrm{~kg}$ (moyenne : 14,2 $\pm 1,6$ ) et $b_{\mathrm{jkl}}$ de 0,187 à 0,487 (moyenne $0,348 \pm 0,083$ ). Calculés à partir du profil horaire $(8$ observations), $A_{j k l}$ a varié de 11,3 à $17,7 \mathrm{~kg}$ (moyenne : 14,0 $\pm 1,6$ ) et $b_{j k l}$ de 0,201 à 0,476 (moyenne $0,346 \pm 0,073$ ). Et finalement, $A_{j k l}$ et $b_{j k l}$ calculés à partir des 4 observations du profil nycthéméral ont varié de 11,7 à $19,5 \mathrm{~kg}$ (moyenne : 14,8 $\pm 1,7$ ) et de 0,173 à 0,437 (moyenne 0,302 \pm 0,072 ). Quel que soit le nombre d'observations, les coefficients sont semblables $(P>$ 0,05).

Par rapport aux coefficients estimés à partir de 1440 observations, les coefficients obtenus à partir du profil horaire varient de $5,5 \%\left(A_{j k}\right)$ à $9,9 \%\left(b_{j k}\right)$. L'erreur de mesure des coefficients calculés avec le profil nycthéméral (4 observations) a été très semblable à celle obtenue avec le profil horaire, c'est-à-dire $5,8 \%$ de $A_{j k l}$ et de $11,2 \%$ de $b_{j k \mid}$. 


\section{Capacité d'ingestion}

Pendant la phase 1, la capacité d'ingestion des vaches, c'est-à-dire l'ingestion de MS à la fin du profil nycthéméral de référence s'élève à $23,2 \pm 1,6 \mathrm{~kg}$, ce qui représente $10,0 \pm 0,5 \mathrm{~kg}$ de constituants pariétaux NDF. Le temps consacré à la prise alimentaire totalise $290 \pm 28 \mathrm{~min}$ et les vaches ont mastiqué $34500 \pm 8300$ fois pendant la rumination $(\mathrm{MM})$ et $3150 \pm 1183 \mathrm{MM}$ $\mathrm{kg}^{-1}$ de constituants pariétaux NDF ingéré. La capacité d'ingestion varie d'une vache à

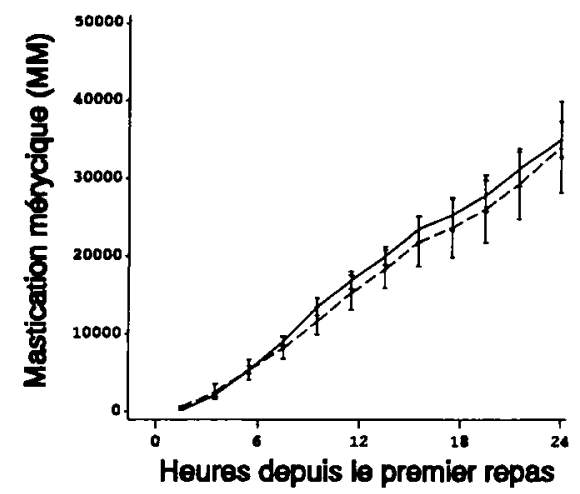

I I Moyennes et Gcarts type du premier carre lath (phase 1)

$f-t$ Moyennes et ccarts type du deuxlème carre latin (phase 1)
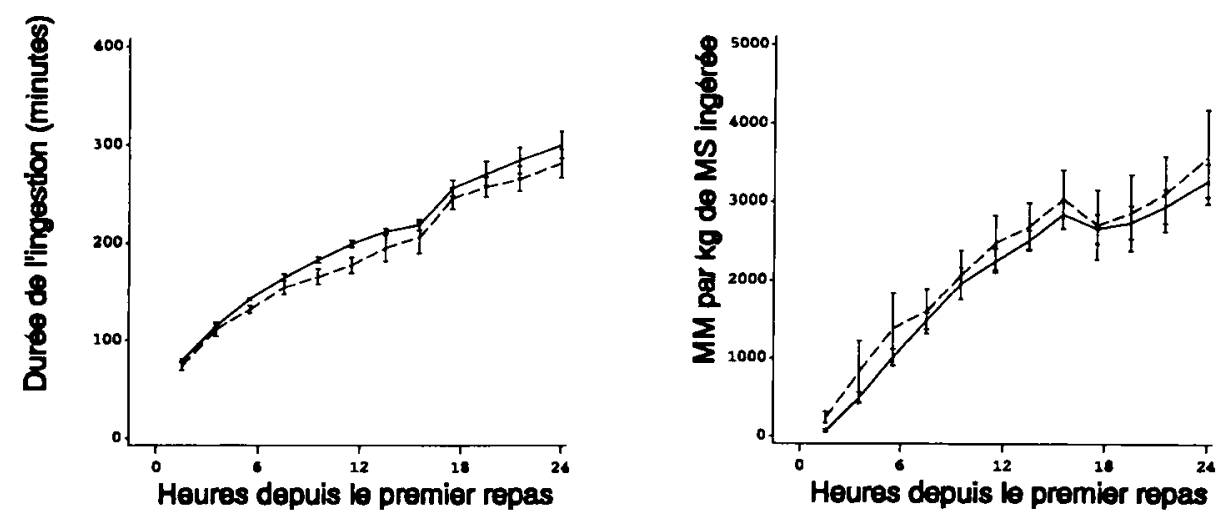

Fig 3. Profils de référence établis pendant la phase 1 pour chacun des carrés latins. l'autre $(P<0,01)$, ainsi que MM $(P<0,05)$ et $\mathrm{MM}$ par $\mathrm{kg}$ de constituants pariétaux NDF $(P<0,05)$, mais pendant la phase 1 , il n'y a aucune corrélation $(P>0,05)$ entre la mastication et la capacité d'ingestion. En conséquence, les profils d'ingestion de MS et de la durée de l'ingestion varient selon le carré latin mais les profils de MM et de $M M k^{-1}$ de constituants pariétaux ne sont pas différents (fig 3 ).

En phase 2, le comportement alimentaire observé avec la ration témoin est identique à celui de la phase 1 (fig 4).

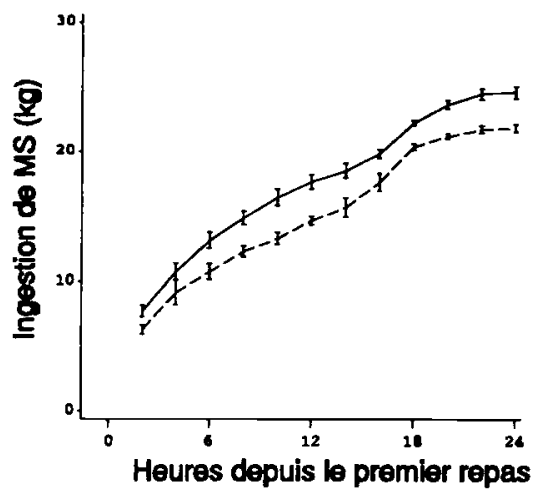




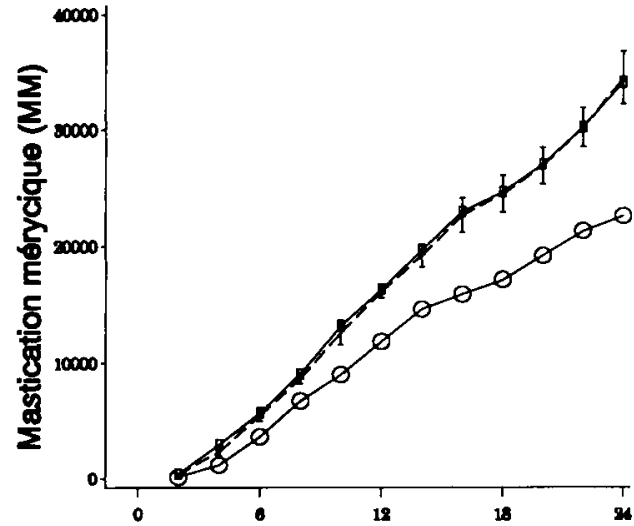

Heures depuis le premier repas

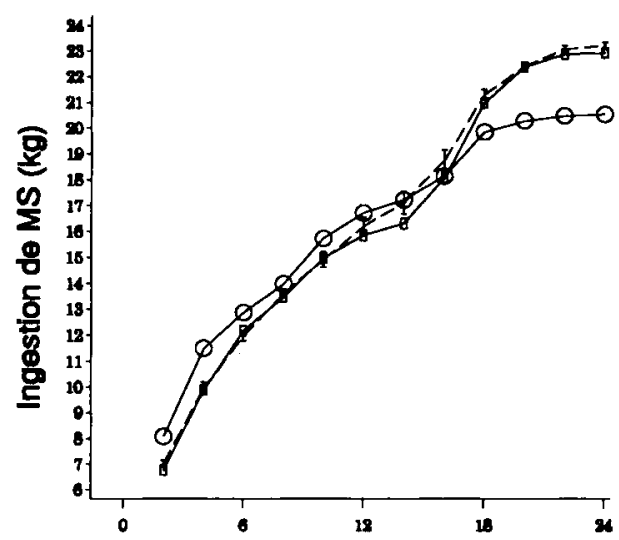

Heures depuis le premier repas

\section{f - $f$ Moyennes et écarts type du témoin (phase 1) \\ Q $\quad$ Q Moyennes du témoin 6 jours plus tard (phase 2) \\ $\ominus$ ○ Moyennes après 6 jours de traitement (phase 2)}

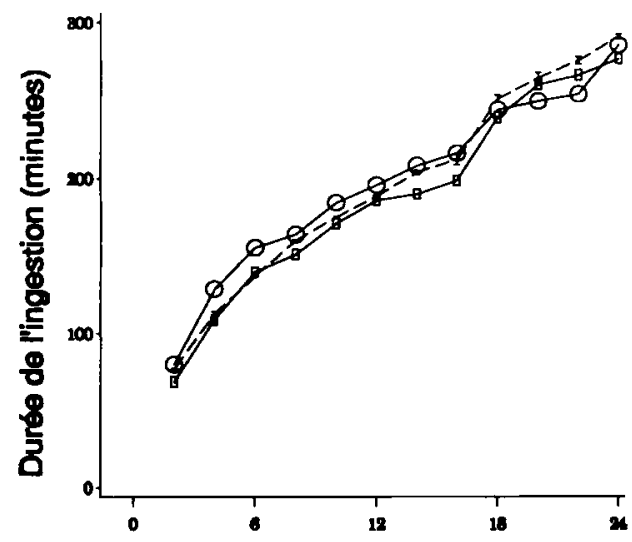

Heures depuis le premier repas

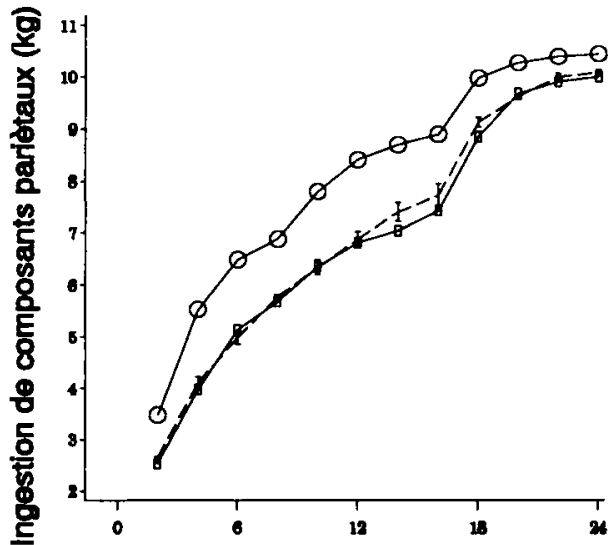

Heures depuis le premler repas

Fig 4. Moyennes des profils de référence (phase 1) et des profils observés 6 j plus tard (phase 2), avec ou sans une modification de l'alimentation. 
Comme le supplément de constituants pariétaux NDF réduit $(P<0,01)$ la capacité d'ingestion $(-10,3 \%)$ et MM $(-33 \%)$ sans affecter l'ingestion de NDF, la modification de la capacité d'ingestion en phase 2 est significativement corrélée à MM et $\mathrm{MM} \mathrm{kg}^{-1}$ de constituants pariétaux (tableau II). L'ingestion de constituants pariétaux NDF et la durée de l'ingestion $4 \mathrm{~h}$ après le début du premier repas sont inversement proportionnelles à la capacité d'ingestion ( $r=-$ 0,66 et $r=-0,52$; tableau II), c'est-à-dire qu'une ingestion de constituants pariétaux plus importante en début de profil se traduit, $20 \mathrm{~h}$ plus tard, par une réduction de la capacité d'ingestion.

L'effet des traitements sur le profil nycthéméral de ces activités et sur celui de l'ingestion de MS est ensuite analysé.

\section{Profils nycthéméraux de référence et variations dues aux traitements}

La dissociation de la variation en contrastes orthogonaux met en évidence l'effet des heures et l'effet de l'interaction heure $x$ vache sur le profil nycthéméral de référence (phase 1). Ces effets sont décrits à l'aide de polynômes du quatrième degré. Quatre observations suffisent donc pour décrire les variations des profils individuels dans nos conditions expérimentales, c'est-à-dire lorsque les vaches sont alimentées une fois par jour en début d'après-midi. Par rapport au traitement témoin, le traitement T + NDF réduit l'ingestion de MS et la mastication mérycique de façon linéaire dans le temps (fig $4, P<$ 0,01 ) tandis qu'il modifie l'ingestion de constituants pariétaux de façon quadratique (fig $4, P<0,01$ ). Sans supplément de constituants pariétaux, les vaches ingèrent en 2 h 7 kg de MS (fig 4). Avec le supplément, elles consomment davantage de MS dans la même période $(+1,1 \mathrm{~kg}$ de MS, $P<$ 0,01 ) et de constituants pariétaux. Cette dernière différence est maximale $14 \mathrm{~h}$ après le premier repas $(+1,7 \mathrm{~kg}$ de constituants pariétaux NDF, $P<0,01)$, c'est-àdire vers $4 \mathrm{~h}$ du matin. Elle est négligeable $(P>0,05) 6 \mathrm{~h}$ plus tard jusqu'au repas suivant (fig 4). Par rapport à la phase 1, le traitement $T+N D F$ a aussi augmenté le temps consacré à l'ingestion en début de profil mais la différence entre les traitements (14 minutes, tableau III) n'est pas significative.

L'analyse de variance des modifications du comportement alimentaire est résumée dans le tableau III. Les coefficients de variation obtenus dans cette analyse sont

Tableau II. Coefficients de corrélation entre les modifications du comportement alimentaire et celles de la capacité d'ingestion.

Comportement alimentaire modifié

\begin{tabular}{|c|c|c|c|}
\hline \multirow{2}{*}{ Comportement alimentaire modifié } & \multicolumn{3}{|c|}{ Heure de lobsenvation dans le profil nyctheméra. } \\
\hline & $2 h$ & $4 h$ & $24 h$ \\
\hline Ingestion de constituants pariétaux & NS & $-0,66^{\star \star \star \star}$ & NS \\
\hline Temps consacré à l'ingestion & NS & $-0,52^{\star}$ & NS \\
\hline Mastication mérycique (MM) & NS & $0,53^{*}$ & $0,76^{\star \star \star}$ \\
\hline MM par kg de constituants pariétaux & NS & $0,65^{\star * *}$ & $0,65^{\star * *}$ \\
\hline
\end{tabular}




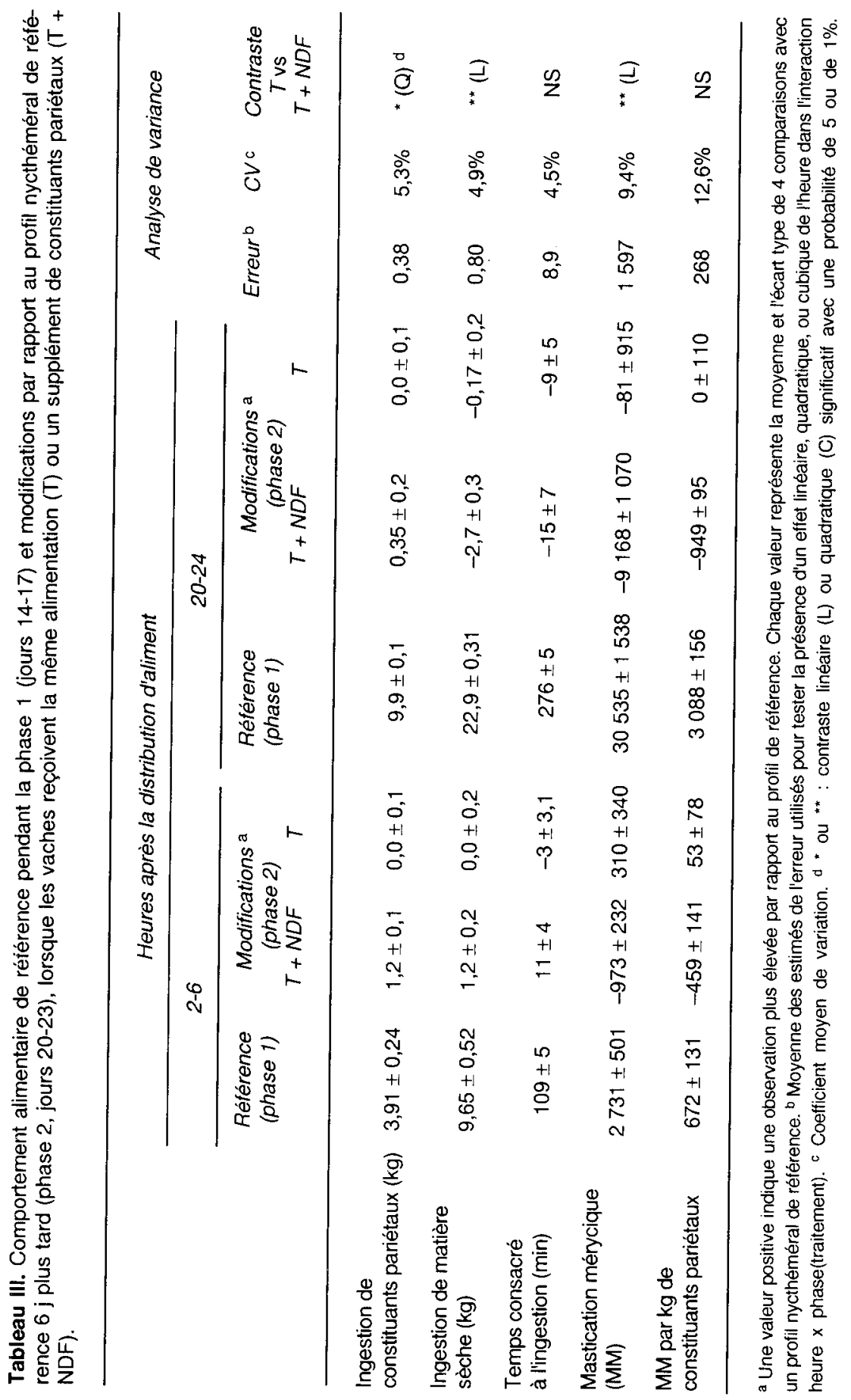


semblables à ceux représentant l'intravariabilité du comportement alimentaire (Girard et Labonté, 1993).

\section{DISCUSSION}

Lorsque la concentration en constituants pariétaux NDF excède $32 \%$, la prise alimentaire dépend de la capacité d'ingestion (Hoover, 1986). Dans cette expérience, les vaches étaient alimentées une fois par jour avec une ration contenant $43,4 \%$ ou $50,7 \%$ de NDF selon le traitement, ce qui garantissait une réplétion maximale du réticulo-rumen. Idéalement, la capacité d'ingestion est exprimée à l'aide d'une activité du comportement alimentaire qui explique à la fois les variations individuelles de la capacité et l'effet des traitements. Dans cette expérience, aucune des activités étudiées n'explique les variations individuelles mais, pour chaque vache, un plus grand repas en début de profil, c'est-à-dire une ingestion de constituants pariétaux plus importante et qui dure plus longtemps, se traduit $20 \mathrm{~h}$ plus tard par une réduction de la capacité d'ingestion. Dans cette expérience, le temps consacré à l'ingestion ou l'ingestion de constituants pariétaux NDF sont constants et si l'on choisit d'ignorer les variations individuelles, chacune de ces 2 activités peut permettre de prédire la capacité d'ingestion. La première a été d'ailleurs déjà été proposée par Moseley et Manendez (1989) et l'invariabilité du deuxième est conforme au modèle de Mertens (1987).

La relation entre ces activités et la capacité d'ingestion ne peut se faire sans une étude plus fine du comportement alimentaire telle que nous la proposons avec l'analyse des profils nycthéméraux. Ces derniers résument en 12 observations l'essentiel des variations du comportement alimentaire. En fait, l'erreur introduite par une telle simplification ne représente que 6-
$11 \%$ des coefficients obtenus lorsque toutes les observations sont utilisées. II est possible de décrire, à l'aide de contrastes polynomiaux, les différences entre vaches ou entre traitements.

Dans cette expérience, la relation entre la capacité d'ingestion et les constituants pariétaux ne peut pas être envisagée comme un ajustement du stimulus qui, en déclenchant la rumination, abrège la durée du repas principal. En effet, le supplément de constituants pariétaux réduit la capacité d'ingestion, malgré un repas principal plus important. II est probable que le mérycisme traduise la qualité des constituants pariétaux dans le rumen, tandis que l'importance du repas principal reflète la valeur d'encombrement des aliments. En fait, ce n'est que 8-12 h après le début du premier repas que le supplément de constituants pariétaux ingéré en début de profil cause un état de réplétion du réticulorumen (Dulphy et Faverdin, 1987) et que les signaux générés (Forbes, 1980) réduisent la prise alimentaire.

Dans cette expérience, la valeur d'encombrement des constituants pariétaux est semblable lorsqu'on se base sur la cinétique ruminale. L'ingestion de constituants pariétaux est bien corrélée en début de profil avec la capacité d'ingestion (tableau II). Mais la différence entre traitements diminue linéairement (tableau III) et, en fin de profil, il n'y a plus de différence entre les traitements. Dans ces conditions, la capacité d'ingestion semble dépendre davantage de la concentration en constituants pariétaux que de l'importance du repas principal ou du temps consacré à l'ingestion. Ce dernier peut d'ailleurs varier sans que cela n'affecte l'ingestion de constituants pariétaux NDF (Kaiser et Combs, 1989).

Dans cette expérience, les vaches consomment $23,2 \mathrm{~kg}$ d'une ration contenant $43,4 \%$ de constituants pariétaux NDF, 
soit, exprimée en constituants pariétaux, $10 \mathrm{~kg}$. En supposant l'invariabilité de la capacité ruminale lorsque les vaches consomment le traitement T + NDF $(50,7 \%$ de constituants pariétaux NDF), l'ingestion volontaire prévue est de $19,7 \mathrm{~kg}(10 / 0,507)$, soit une réduction de la capacité d'ingestion équivalente à $3,5 \mathrm{~kg}$. La réduction prévue est supérieure à celle observée (tableau (II), mais la différence peut être imputable à la réduction de la rumination concomitante au traitement T + NDF. Pendant la rumination, le taux de passage horaire moyen des petites particules diminue (Girard, 1990) et la valeur d'encombrement basée sur la cinétique ruminale augmente. La relation entre la mastication mérycique (MM) et MM par kg de composants pariétaux et la capacité d'ingestion (tableau II) s'explique, selon nous, par l'effet de la rumination sur la valeur d'encombrement.

\section{CONCLUSION}

Le dispositif statistique que nous proposons permet de décrire à l'aide de 11 contrastes polynomiaux l'effet du moment de la journée sur le comportement alimentaire d'une vache recevant plus ou moins de constituants pariétaux dans sa ration. Cette expérience confirme l'existence d'un lien entre la durée ou l'importance des repas qui suivent la distribution d'aliment à l'auge et la capacité d'ingestion (Nørgaard, 1986 ; Deswysen et al, 1987 ; Baumont et al, 1989 ; Moseley et Manendez, 1989 ; Francke et al, 1990) ; $24 \mathrm{~h}$ après le début du premier repas, 2 paramètres du comportement alimentaire ne sont pas affectés par les traitements : le temps consacré à l'ingestion et l'ingestion de constituants pariétaux. La capacité d'ingestion, exprimée en constituants pariétaux, sous-estime de $0,5-0,7 \mathrm{~kg}$ la prise alimentaire de MS lorsque les vaches reçoivent le traitement $T+$
NDF. Cette différence peut cependant être expliquée par la réduction de la rumination, qui coïncide avec l'introduction de constituants pariétaux de faibles dimensions dans le traitement T + NDF.

\section{REMERCIEMENTS}

Nous remercions $R$ Grégoire et $M$ Côté pour leur aide dans la réalisation de cette expérience.

\section{RÉFÉRENCES}

Beauchemin KA, Buchanan-Smith JG (1989) Effects of dietary neutral detergent fiber concentration and supplementary long hay on chewing activities and milk production of dairy cow. J Dairy Sci 72, 2288-2300

Baumont R, Brun JP, Dulphy JP (1989) influence of the nature of hay on its ingestibility and the kinetics of intake during large meals in sheep, cows. In: XVI International Grassland Congress, Nice

Baumont R, Malbert CH, Ruckebusch Y (1990) Mechanical stimulation of rumen fill and alimentary behavior in sheep. Anim Prod 50, 123-128

Charmley E, Veira DM, Butler G, Aroeira L, Codagnone CV (1991) The effect of frequency of feeding and supplementation with sucrose on ruminal fermentation of alfalfa silage given ad libitum or restricted to sheep. Can J Anim Sci 71, 725-737

Dehareng D, Godeau JM (1991) The durations of masticating activities and the feed energetic utilisation of friesian lactating cows on maize silage-based rations. I Anim Physiol Anim Nutr 65, 194-205

Deswysen AG, Ellis WC, Pond KR (1987) Interrelationships among voluntary intake, eating, ruminating behavior, ruminal motility of heifers fed corn silage. J Anim Sci 64, 835-841

Dulphy JP, Carle B, Demarquilly C (1990) Quantités ingérées et activités alimentaires comparées des ovins, bovins et caprins recevant des fourrages conservés avec ou sans aliment concentré. 1. Étude descriptive. Ann Zootech 39, 95-111 
Dulphy JP, Faverdin P (1987) L'ingestion alimentaire chez les ruminants: modalité et phénomènes associés. Reprod Nutr Dév 27 , 129-155

Forbes JM (1980) A model of short-term control of feeding in the ruminant: effects of changing animal or feed characteristics. Appetite 1, 21-41

Francke H, Junge W, Kalm E (1990) Roughage intake of dairy cows with special consideration of the behavior. 2. Communication. Equations for the estimation of the roughage intake. Züchtungskunde 62, 468-480

Girard V (1990) Effect of rate of intake on ruminal rate of passage of small forage particles. Can J Anim Sci 70, 243-250

Girard V, Labonté J (1993) Dispositif d'enregistrement en continu du comportement alimentaire journalier et étude de la variabilité du comportement alimentaire chez la vache laitière. Ann Zootech 42, 49-59

Girard V, Pelletier G (1987) Interpretation of non-steady-state rumen liquid marker dilution curves. Can J Anim Sci 67, 563-567

Hoover WH (1968) Chemical factors inolved in ruminal fiber digestion. J Dairy Sci 69, 2755

INRA (1978) Le système des unités d'encombrement pour les bovins. In: Alimentation des ruminants (R Jarrige, ed). Versailles, France
Jarrige R, Demarquilly C, Dulphy JP et al (1986) The INRA 'fill unit' system for predicting the voluntary intake of forage-based diets in ruminants: a review. J Anim Sci63, 1737-1758

Kaiser RM, Combs DK (1989) Utilization of three maturities of Alfalfa by dairy cows fed rations that contain similar concentrations of fiber. J Dairy Sci 72, 2301-2307

McLeod MN, Minson J (1988) Large particle breakdown by cattle eating rye-grass and alfalfa. J Anim Sci 66, 992-999

Mertens DR (1987) Predicting intake and digestibility using mathematical models of ruminal function. J Anim Sci 64, 1548-1558

Moseley G, Manendez AA (1989) Factors affecting the eating rate of forage feeds. $I n: X V \mid$ International Grassland Congress, Nice

Norgaard P (1986) Influence of the physical form of diet on chewing activity and reticulo-rumen motility in cows. Acta Vet Scand 86, 46-52

Rowell JG, Walters DE (1976) Analysing data with repeated observations on each experimental units. J Agric Sci Camb 87, 423-432

SAS Institute Inc (1987) SAS/STAT Guide for personal computers, version 6 edition SAS Institute Inc

Van Milgen J, Murphy MR, Berger LL (1991) A compartmental model to analyse ruminal digestion. J Dairy Sci 74, 2515-2529 